\title{
Polymer-Brush Lubrication in Oil Sliding Beyond the Stribeck Curve
}

\section{Journal Article}

Author(s):

Bielecki, Robert M.; Crobu, Maura; Spencer, Nicholas D.

Publication date:

2013-01

Permanent link:

https://doi.org/10.3929/ethz-b-000062805

\section{Rights / license:}

In Copyright - Non-Commercial Use Permitted

Originally published in:

Tribology Letters 49(1), https://doi.org/10.1007/s11249-012-0059-9 


\title{
Polymer-Brush Lubrication in Oil: Sliding Beyond the Stribeck Curve
}

\author{
Robert M. Bielecki • Maura Crobu • \\ Nicholas D. Spencer
}

Received: 24 July 2012/ Accepted: 10 October 2012/Published online: 4 November 2012

(C) Springer Science+Business Media New York 2012

\begin{abstract}
We report a highly effective macroscopic, nonaqueous lubrication strategy, employing polymer-brushbased surface modification. Poly(alkyl methacrylates) with hexyl, dodecyl and octadecyl side chains have been prepared on both silicon and iron substrates by means of surface-initiated atom-transfer radical polymerisation. Frictional properties of the three polymers have been evaluated in hexadecane, ethanol and toluene. For the case of the poly(dodecyl methacrylate), the study additionally involved nine base lubricating fluids of different chemical natures, with viscosities ranging from 4 to $2,200 \mathrm{cSt}$ at $20{ }^{\circ} \mathrm{C}$. By constructing a set of Stribeck curves at low values of speed $\times$ viscosity (at constant load), it was possible to identify an extended hydrodynamic lubrication regime and, for P12MA-based coatings, a low-friction, brush-lubrication regime, reinforced by time-dependant effects, which appeared to replace the mixed and boundary regimes observed for conventional lubricants. Self-mated poly(dodecyl methacrylate) brushes maintained low coefficient-of-friction values (e.g. 0.012 in hexadecane) over thousands of reciprocating cycles ( $>100 \mathrm{~m}$ total distance) under low speed $\times$ viscosity conditions $(0.1 \mathrm{~cm} / \mathrm{s}$ and $4 \mathrm{cSt})$.
\end{abstract}

Keywords Polymer brush - Surface modification . ATRP · Oil lubrication · Viscosity - Steel · Lubrication regimes $\cdot$ Stribeck curve

Electronic supplementary material The online version of this article (doi:10.1007/s11249-012-0059-9) contains supplementary material, which is available to authorized users.

R. M. Bielecki · M. Crobu · N. D. Spencer $(\bowtie)$ Laboratory for Surface Science and Technology, Department of Materials, ETH Zurich, Wolfgang-Pauli-Strasse 10, 8093 Zurich, Switzerland

e-mail: nspencer@ethz.ch

\section{Introduction}

Lubrication with polymer brushes has been researched intensively in recent years, following the pioneering work of Klein et al. [1, 2]. Many studies of polymer-brush tribology have involved nanoscale measurements carried out by means of SFA [3-5] or AFM [6-8]. A comparatively smaller amount of work has been carried out on a macroscopic scale, investigating friction dependence of grafting density [9], solvent quality and swelling states [10,11], for example.

Developments in polymer chemistry, especially in controlled radical polymerisation [12, 13], have increased interest in the tribological properties of polymer brushes. Studies have frequently concerned lubrication in water or common organic solvents, such as hexane or toluene. The effects of lubricant viscosity, investigated by means of AFM, were reported by Nomura et al. [14] when studying poly(methyl methacrylate) (PMMA) brushes in ionic liquids. We recently reported an atom-transfer radical polymerisation (ATRP)-based approach to prepare poly(alkyl methacrylate) brushes, which showed very low friction in a non-aqueous medium: By means of lateral-force microscopy and preliminary macrotribological tests, we were able to demonstrate the potential of such bottle-brush-like, surfacetethered molecules for achieving low-friction conditions, using hexadecane as a model lubricant [15].

In the present study, we have investigated in detail the tribological behaviour of a series of polymer brushes consisting of methacrylates substituted with long alkyl side chains, synthesized via surface-initiated, atom-transfer radical polymerisation (SI-ATRP) ('grafting-from' approach). The tribological properties of polymer brushes synthesized from hexyl-, dodecyl- and octadecyl methacrylate monomers (named P6MA, P12MA and P18MA, respectively) were evaluated by means of a tribometer covering the 
0.001-0.1 $\mathrm{N}$ range in the presence of base fluids of different chemical natures and viscosities. Maximal Hertzian pressures $>200 \mathrm{MPa}$ could readily be achieved between countersurfaces consisting of a flat silicon wafer and a borosilicate glass ball ( $2 \mathrm{~mm}$ in diameter). The countersurfaces were both coated with the polymer brushes ('brush-brush') and the tribological results compared to those for which both countersurfaces were polymer free ('bare-bare').

Silicon has typically been the substrate of choice for most SI-ATRP studies, while steel is clearly of greater industrial interest. However, there are only a few reports of grafting from polymer-brush strategies involving ironbased substrates in the literature [16]. Lu et al. [17] applied SI-ATRP to form corrosion-resistant PMMA coatings on iron coupons. Fan et al. [18] used ATRP with a biomimetic (catecholamine-based) anchoring group to form non-fouling PEG brushes on 316L steel surfaces. The present study includes the synthesis and tribological evaluation of poly(dodecyl methacrylate) brushes on iron oxide and steel surfaces. The presence of iron introduces additional complications, since the Cu-based ATRP catalyst may undergo reduction and lose its activity during the reaction [19]. For this reason, the previously reported protocol for poly(alkyl methacrylate) synthesis via SI-ATRP has been modified, and a nitrodopamine-based anchoring group has been introduced [20] for use with iron-containing substrates.

\section{Experimental}

\subsection{Surfaces and Their Functionalisation}

One side-polished silicon wafers ( $\mathrm{P} / \mathrm{B}<100>$, Si-Mat Silicon Wafers, Germany) were functionalised with 11-(2-bromo2-methyl-propionyl)-dimethylchlorosilane (BPCS) initiator molecules, synthesized according to Sanjuan et al. [21], following our previously described surface-deposition protocol [15]. Borosilicate glass balls (Hauser Optik, Germany), $2 \mathrm{~mm}$ in diameter, were treated in a similar way to the flat silicon wafers. In order to reduce discrepancies between coatings produced on the two substrate shapes, for any given coating, balls and wafers were always functionalised simultaneously and the polymerisation reaction was carried out in the same reaction vessels, according to our previously described procedure.

Briefly, a solution of the monomer in anisole (10 vol\%), together with 4,4'-dinonyl-2,2'-bipyridine (Aldrich Fine Chemicals, $97 \%$ ), underwent four freeze-pump-thaw cycles to remove oxygen. The prepared solution was transferred to a flask containing $\mathrm{Cu}(\mathrm{I}) \mathrm{Br}$ and $\mathrm{Cu}(\mathrm{II}) \mathrm{Br}_{2}$ salts and left at elevated temperature, in order to achieve a homogenous, dark-brown mixture. This monomer mixture was dispensed onto the BPCS-functionalised substrates, which were placed in a glass container and kept in an argon-filled glove box $\left(\mathrm{O}_{2}\right.$ level $\left.<200 \mathrm{ppm}\right)$ at $110^{\circ} \mathrm{C}$. After this, the reaction was left for the desired time until quenched by dilution in toluene and exposure to air. During the reaction, the ratio of monomer:ligand:copper(I) was maintained at 150:1.5:1 with a $15 \%$ proportion of $\mathrm{Cu}(\mathrm{II})$ to $\mathrm{Cu}(\mathrm{I})$ salts. After synthesis, samples were washed multiple times with toluene, isopropanol and a $0.1 \mathrm{M}$ solution of $\mathrm{Na}_{2}$ EDTA to remove copper moieties (Fig. 1).

Iron(III) oxide coatings were deposited on polished silicon wafers by means of magnetron sputtering at the Paul Scherrer Institut, Villigen, Switzerland. Polished 100Cr6 (AISI 52100) steel balls, $2 \mathrm{~mm}$ diameter, were purchased from CSM Instruments (Peseux, Switzerland). Surface functionalisation of iron oxide-coated wafers and steel balls with initiator molecules was carried out by means of a protocol similar to that applied for silicon-wafer substrates. First, substrates were sonicated three times with 2-isopropanol and treated for 30 min in a UV Ozone cleaner (UV/Ozone ProCleaner ${ }^{\mathrm{TM}}$, BioForce, IA, USA). The cleaned samples were incubated overnight in a solution of in-house-synthesized nitrodopamine [20] solution $(50 \mu \mathrm{g} / \mathrm{ml}$ in 1:39 dimethylformamide:ethanol mixture), after which the samples were washed with ethanol and dried. Subsequently, samples were placed for $2 \mathrm{~h}$ in dichloromethane $(50 \mathrm{ml})$, to which triethylamine $(0.8 \mathrm{ml})$ and bromo-isobutyrate bromide $(0.8 \mathrm{ml})$ had been added. Afterwards the samples were washed with dichloromethane, then with isopropanol and dried.

Due to potential interactions of the iron-based surfaces with copper-based complexes used for silicon functionalization, the SI-ATRP protocol for the synthesis of dodecyl methacrylate was modified and iron bromide(II) $(99,999 \%$, Aldrich Fine Chemicals), iron bromide(III) (98\%, Aldrich Fine Chemicals) and triphenyl phosphine ( $>98.5 \%$, Fluka) were used as activating, deactivating and ligand species, respectively. The monomer:ligand: $\mathrm{FeBr}_{2}$ molar ratio was adjusted to 300:3:1 and 10 vol\% of tetrahydrofuran was added as a solvent.

A variable-angle spectroscopic ellipsometer (M-2000F, LOT Oriel GmbH, Darmstadt, Germany) was used to determine the initiator deposition efficiency and the dry thickness of the surface-bound polymers. The wavelength range for which fitting was performed lay between 370 and $995 \mathrm{~nm}$ and the refractive index of the coating was assumed to be 1.45 .

\subsection{Lubricating Fluids}

In addition to ethanol (kinematic viscosity ca. $1.52 \mathrm{cSt}$ ), toluene (kinematic viscosity ca. $0.68 \mathrm{cSt}$ ) and hexadecane (kinematic viscosity ca. $4 \mathrm{cSt}$ ), commercial, additive-free lubricating fluids characterised by kinematic viscosities ranging from 36 to $2,200 \mathrm{cSt}$ at $20{ }^{\circ} \mathrm{C}$ and of two general types were used (petroleum fractions $P F$ and ester oils $E O$, listed in Table 1). 
Fig. 1 Schematic representation of surface modifications: a applied to the silicon substrates and $\mathbf{b}$ to iron oxide substrates. The binding interactions are simplified for clarity. Dodecyl methacrylate is shown as the monomer (a)
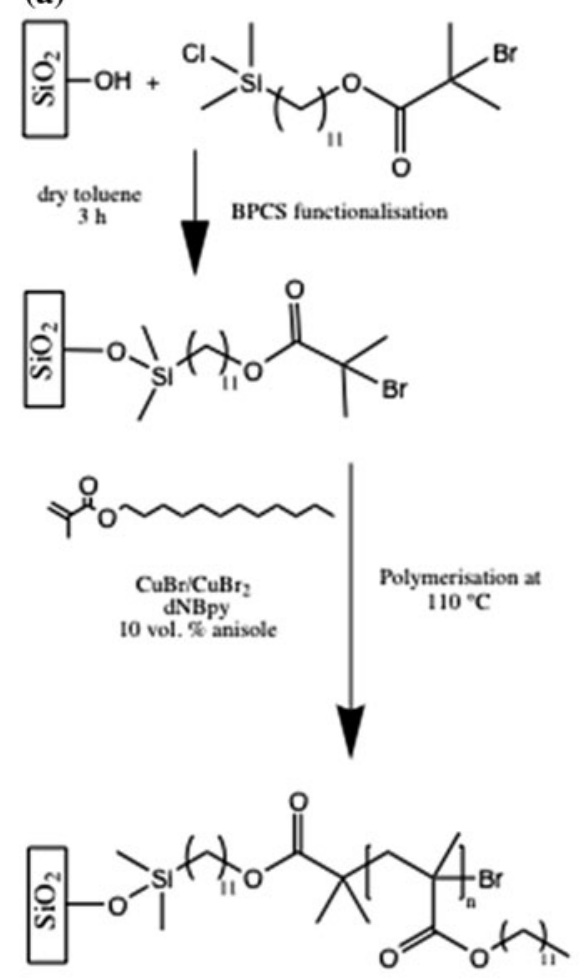

(b)

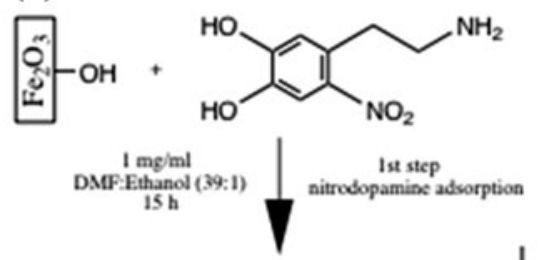<smiles>CC(C)(Br)C(=O)Br</smiles>

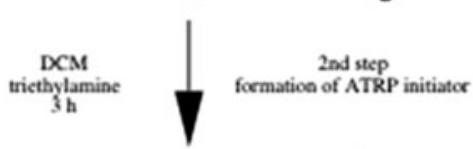<smiles></smiles>

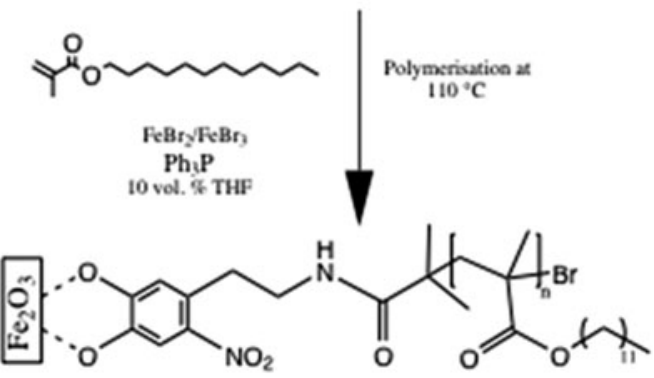

Table 1 Viscosity values and sources of the oils used in this study

\begin{tabular}{|c|c|c|c|}
\hline Name & $\begin{array}{l}\text { Kinematic } \\
\text { viscosity at } \\
20{ }^{\circ} \mathrm{C}(\mathrm{cSt})\end{array}$ & Origin & Common name \\
\hline PF36 & 36 & $\begin{array}{c}\text { Brenntag AG, } \\
\text { Switzerland }\end{array}$ & Vaseline oil \\
\hline PF350 & 350 & $\begin{array}{c}\text { ENI Suisse SA, } \\
\text { Switzerland }\end{array}$ & Neutral oil \\
\hline PF950 & 950 & $\begin{array}{l}\text { (Motorex AG/ } \\
\text { Brenntag AG) }\end{array}$ & $\begin{array}{l}85 \% \\
\text { Brightstock/ } \\
15 \% \text { Vaseline } \\
\text { oil }\end{array}$ \\
\hline PF2200 & 2,200 & $\begin{array}{r}\text { Motorex AG, } \\
\text { Switzerland }\end{array}$ & Brightstock \\
\hline EO500 & 500 & $\begin{array}{l}\text { Erbslöh AG, } \\
\text { Switzerland } \\
\text { (Croda, } \\
\text { Uniquema), }\end{array}$ & Polyol ester \\
\hline EO1300 & 1,300 & $\begin{array}{l}\text { Erbslöh AG, } \\
\text { Switzerland } \\
\text { (Croda, } \\
\text { Uniquema), }\end{array}$ & Polyol ester \\
\hline
\end{tabular}

Before starting tribological experiments, a few drops of lubricant were deposited on a disc, so as to cover the entire intended sliding track, after which a ball was brought close to the surface, allowing a meniscus to be established. Such evenly wetted samples were allowed to equilibrate with the solvent for $10 \mathrm{~min}$ prior to the application of contact pressure.

\subsection{Tribological Studies}

Tribological studies were performed with a NTR2 tribometer (CSM Instruments, Peseux, Switzerland) in a ball-on-disc configuration. For the purpose of friction measurement, the lateral displacement is detected via capacitance measurements, and converted to a lateral-force signal. The normal force is applied via a piezo-actuator controlled within a servo loop. In this arrangement, the capacitive sensor allows the actual normal force to be continuously monitored and compared with the nominal force, any discrepancies being immediately corrected by means of piezoelectric-based cantilever-height adjustment. The relative nominal amplitude of the load error remained below $0.05 \%$. This approach to normal-force control allows the uncertainty parameter related to variable contact pressure to be essentially eliminated. Note that during the tribological test, the coefficient of friction is calculated as the ratio of the values of current lateral force and current normal load (i.e. assuming Amontons' law to hold). 
All tests were carried out in rotating reciprocating mode, over an arc of $120^{\circ}$ and with the speed changing sinusoidally. Such a configuration has several advantages: (a) it is bidirectional, and thus the systematic error related to the misalignment of the samples is eliminated [22], (b) any error related to frictional-force drift can be easily eliminated by subtracting measured friction values recorded during clockwise and counterclockwise motion and (c) the sinusoidal motion allows the friction to be measured as a function of both speed and direction.

Stribeck-like curves were collected at constant load, starting at high sliding speed $(5 \mathrm{~cm} / \mathrm{s})$, then moving to successively lower sliding speeds, through the lowest sliding speed $(0.01 \mathrm{~cm} / \mathrm{s})$, after which the sliding speeds were increased again, back to the highest sliding speed $(5 \mathrm{~cm} / \mathrm{s})$. At least 20 cycles were completed at each speed. After each set of measurements at a given speed, the tribometer was stopped, and the load was removed and reapplied before commencing sliding at another sliding-speed value.

The sliding-speed values reported in this study relate to the maximal values attained within the (sinusoidal) reciprocating cycle. For the purposes of data analysis, at each sliding speed $v$ and for each reciprocating cycle $C_{v}$, data points were sorted into separate matrices for clockwise $(\mathrm{CW}) M_{c v+}$ and counterclockwise $(C C W) M_{c v-}$ motion. Within each such matrix, only the centrally located $30 \%$ of data points (for which the actual sliding speed varied between 90 and $100 \%$ of the maximal nominal sliding speed) were used and averaged separately.

In the case of stability studies (Figs. 4, 5, 6, 7), the coefficient of friction is presented for each cycle separately, in order to enable precise tracking of its variation. For a single cycle the value was obtained as:

\section{Results and Discussion}

3.1 Polymer Brushes of Hexyl-, Dodecyland Octadecyl Methacrylates Studied in Ethanol, Toluene, Hexadecane and PF350 Oil

Silicon wafers and borosilicate glass balls in a bare-bare or brush-brush configuration were studied under $20 \mathrm{mN}$ load and $0.1 \mathrm{~cm} / \mathrm{s}$ sliding speed, in a dry state under ambient conditions, and also immersed in ethanol, toluene, hexadecane or PF350 oil.

Coefficient-of-friction values obtained while sliding over 20 cycles in the rotating reciprocating motion using bare-bare and brush-brush modified configurations are presented in Fig. 2. Coatings on the P6MA, P12MA and P18MA surfaces were ca 90, 250 and $230 \mathrm{~nm}$ in dry thickness, respectively.

All three polymer brushes, when studied in toluene, maintained a low $C O F$ in the range of $0.01-0.02$ over the entire 20-cycle-testing period and the polymer coatings showed no damage within the contact region. Further, when compared to the bare-bare sliding in toluene, the coatings had reduced the coefficient of friction by a factor of 10. In comparison, the group of Takahara [23] has described the synthesis and macrotribological assessment of poly(hexyl methacrylate) brushes, and $C O F$ values of 0.05 upon toluene were reported.

Grafting density values of grafted-from polymer brushes reported in the literature are $0.6-0.7,0.56$ [23] and 0.27-0.32 chains $/ \mathrm{nm}^{2}$ [24] for PMMA, P6MA and P12MA, respectively. In the case of the poly(alkyl methacrylates) reported here, the different monomer side-chain lengths $(6,12$ and 18) potentially result in different polymer-brush

$C O F_{\text {cycle } i}=\frac{\text { average } C O F \text { in the } C W \text { direction }+ \text { average } C O F \text { in the } C C W \text { direction }}{2}$

In the case of the other tribological results presented, the coefficient-of-friction values were averaged over 20 cycles.

The error bars in the stability studies correspond to the standard deviations of the coefficient-of-friction values that were included in the averaging process. For stability tests, three or more repeats were carried out for each combination of conditions (two if the data reproducibility was clearadditional data is provided in the Supplementary Material).

In the case of the other tribological results, the standard deviation of $C O F$ values from each of the 20 cycles is included additionally. grafting densities $-\sigma$ might be expected to be in the order $\sigma$ P6MA $>\sigma_{\text {P12MA }}>\sigma_{\text {P18MA }}$. According to Perry et al. [25], who studied the influence of $\sigma$ on $C O F$ values in poly (L-lysine)-graft-poly(ethylene glycol) grafted-to brush systems, higher $C O F$ values are generally observed for lower grafting densities. Thus, in the case of P6MA, P12MA and P18MA, a higher COF could be expected for the bulkier monomers, which inhibit dense packing of the polymeric backbones on the surface. However, the fact that for the three coating types the obtained $C O F$ values in toluene were equally low, could be explained using the 


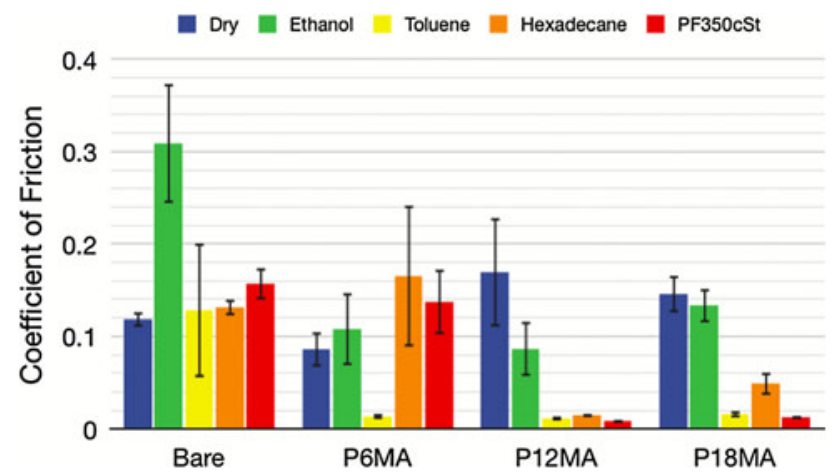

Fig. 2 Coefficient-of-friction values measured at $0.1 \mathrm{~cm} / \mathrm{s}$ for barebare (silicon wafer-borosilicate ball) and polymer-polymer configurations of P6MA, P12MA and P18MA. Data were collected under dry (ambient) conditions, in ethanol, in toluene, in hexadecane and in petroleum fraction oil of $350 \mathrm{cSt}$ viscosity. Values are averaged over 20 reciprocating cycles

procedure suggested by Landherr [9], in which grafting densities, $\sigma$, are additionally divided by the monomer size (to yield a 'reduced grafting density').

It should be noted that, in the case of P6MA, the lower coating thickness did not prevent it from attaining low coefficient-of-friction values in toluene (Fig. 2).

In order to approach the conditions of potential applications of the studied brush systems, experiments were carried out in hexadecane and PF350 hydrocarbon oil. Both the P12MA and P18MA polymers displayed low COFs in both of these model base stocks. In contrast, the P6MA-based brushes did not show low-friction performance in these fluids: Over 20 reciprocating cycles the averaged coefficientof-friction values were greater than 0.1 and complete coating removal was observed by means of optical microscopy.

The longer alkyl-side-chain-substituted polymers (P12MA and P18MA), when studied in PF350, both showed COF values below 0.02 . The use of hexadecane as a lubricating fluid showed slightly higher coefficient-of-friction values for the P18MA (COF $=0.049)$. Nonetheless, both P12MA and P18MA seem to be well suited for lubrication in oils.

Control sliding experiments under dry (ambient) conditions and in ethanol were conducted, for which high COF values were recorded, regardless of the substrate-modification type. For all three polymer coatings, coating removal was observed by optical microscopy within 20 cycles of dry sliding or sliding in ethanol.

\subsection{Effect of Lubricant Type on the Speed Dependence of the $C O F$, for P12MA (brush-brush)}

Figure 3 presents Stribeck-like curves for bare contact pairs and for those modified, on both countersurfaces, with P12MA polymer brushes. The curves were collected under conditions (dry thickness $250 \mathrm{~nm}$ and load of $20 \mathrm{mN}$ )

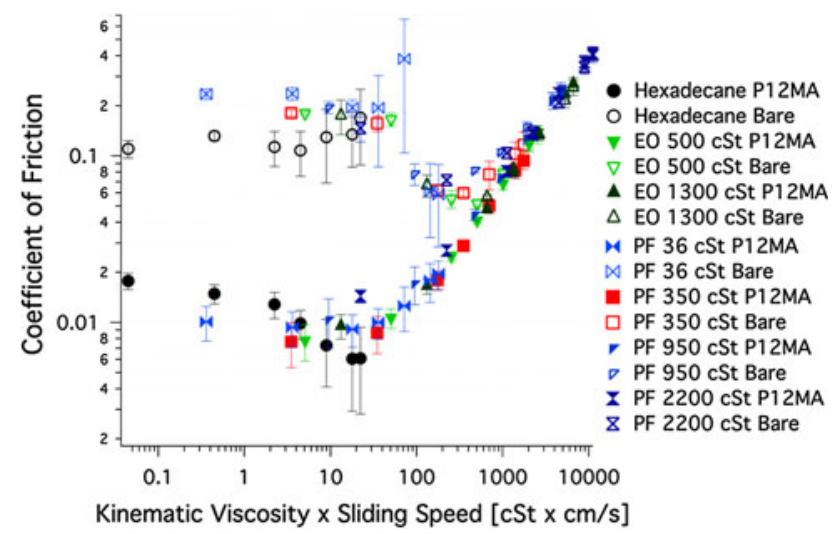

Fig. 3 Stribeck-like plots at constant load $(20 \mathrm{mN})$ obtained for nine different base fluids for bare-bare $\mathrm{SiO}_{2}$-borosilicate glass ball (empty markers) and for double-sided $250 \mathrm{~nm}$ (dry thickness) P12MA brushbrush functionalised tribological contacts (filled markers). Each marker represents the average $\mathrm{COF}$ of 20 reciprocating cycles

under which coating degradation could neither be observed by optical microscopy, nor by tribological measurements.

At high speeds and high viscosities above a certain product of speed $\times$ viscosity, all data points fall on the straight linecharacteristic of hydrodynamic lubrication. Within the transition region in which the hydrodynamic regime normally enters the mixed-lubrication regime (deviation from the straight line), the data separated into two distinct groups, corresponding to bare-bare and brush-brush. The bare-bare cases entered the mixed-lubrication regime in the expected manner, transitioning to boundary lubrication $(\mu>0.1)$ at low speed $\times$ viscosity number. In contrast, the brush-brush samples showed a transition from the straight-line hydrodynamic behaviour, directly to a low and nearly constant friction value $(\approx 0.01)$ at low speed $\times$ viscosity number. This is attributed to the brush-lubrication regime. The specific base stock did not appear to affect this behaviour significantly (all were good solvents for the polymer brush). Interestingly, the transition between mixed and boundary regimes for the barebare system occurred at the same speed $\times$ viscosity value as the hydrodynamic-brush transition for the brush-brush case. The low COF of the brush regime observed in this study (Fig. 3) is similar to that obtained for P12MA when sliding in toluene (Fig. 1) under the conditions when speed $\times$ viscosity equals $0.068 \mathrm{cSt} x \mathrm{~cm} / \mathrm{s}$, corresponding to the far left end of the plot.

The brush-lubrication regime observed in Fig. 3 did not show a significant speed dependence, which is consistent with the experimental AFM work of Nomura et al. [10], who showed speed-invariant $C O F$ values in the low-friction regime for poly(styrene) brushes in toluene (at lower shear velocities), and molecular dynamics simulations by Dai et al. [26], who also showed speed-independent $C O F$ sliding regimes for poly(ethylene) brush-brush systems. 
3.3 The Effect of Polymer Thickness, Sliding Speed and Load on Coating Stability and Lubricious Properties

\subsubsection{Thickness as a Parameter}

Figure 4 presents frictional data obtained in reciprocating motion experiments with $0.1 \mathrm{~cm} / \mathrm{s}$ maximum speed and $20 \mathrm{mN}$ load for silicon wafers and borosilicate glass balls, coated symmetrically with P12MA films of 70,140 and $250 \mathrm{~nm}$ dry thickness and studied in three different lubricants.

Studies conducted in hexadecane as a lubricant revealed that wear resistance decreases with decreasing coating thickness. In particular, 70 and $140 \mathrm{~nm}$-thick coatings lost their low-frictional properties within the first few metres of sliding. In contrast, the thickest coating $(250 \mathrm{~nm})$ was shown to be much more stable, maintaining a $C O F$ below 0.012 for sliding distances over $100 \mathrm{~m}$ and displaying no damage detectable by optical microscopy. This behaviour can be explained in terms of the greater amount of solvated polymer layer per unit area of contact for the thicker brushes. Upon loading, the brush will be compressed, but the higher the initial thickness the greater the ultimate thickness of the cushioning layer between the hard countersurfaces and the lower the likelihood of local, destructively high contact pressures due to roughness effects.

The stability of the $140 \mathrm{~nm}$ coating was significantly improved by a change to a more viscous ester lubricant (EO500). Shortly after the beginning of the tests (ca. 6 cycles or $10 \mathrm{~cm}$ ), sliding behaviour characterised by a low $C O F$ (ca 0.024 ) was established and maintained over $100 \mathrm{~m}$ sliding distance. This stability-enhancement effect was also observed for the thinnest of the analysed coatings (70 nm). Specifically, sliding of $70 \mathrm{~nm}$-thick P12MA brush-brush systems characterised by $C O F \approx 0.03$ was observed for both the EO500 ester lubricant and for a petroleum

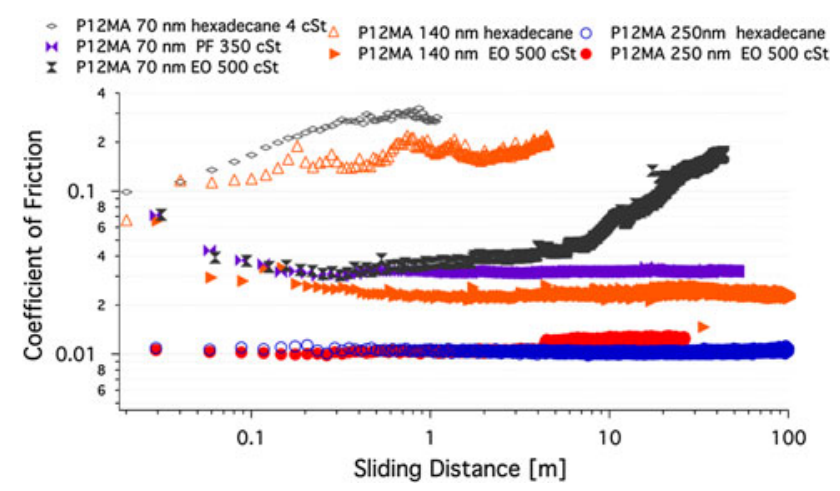

Fig. 4 Coefficient-of-friction evolution as a function of sliding distance between a silicon wafer and borosilicate glass ball, both sides being modified with P12MA brushes. The effect of coating thickness and base fluid on stability is investigated. Data collected at a sliding speed of $0.1 \mathrm{~cm} / \mathrm{s}$ and load of $20 \mathrm{mN}$ fraction-based lubricant of equivalent viscosity (PF350). The achievement of low $C O F$ and improved protection of the coatings against wear, even for thinner polymeric films, could be observed with more viscous lubricants, although their chemical nature also appeared to have an effect on the wear stability of the coating: The more rapid failure of the 70-nm-thick P12MA coating in the EO500 oil (500 cSt), when compared to PF350 (350 cSt), may be attributable to differences in solvent quality.

The P12MA coating thickness appeared to have an effect on the $C O F$ values during sliding. $C O F$ values for 140 and 70-nm-thick P12MA brushes slid in EO500 were higher than that of the thickest coating $(250 \mathrm{~nm})$, suggesting that thicker, higher molecular-weight brushes lead to lower $C O F$ values. This is consistent with previous, sharp-tip, lateral-forcemicroscopy studies by Zhang et al. [27], who found that poly(2-(methacryloyloxy)ethylphosphorylcholine) brushes immersed in aqueous solutions showed lower coefficients of friction at greater brush lengths. Sakata et al. [28], using macroscopic pin-on-disc measurements, showed that under good solvent conditions (acetone and toluene), the thicker the PMMA brushes, the lower the $C O F$ values. The literature examples, as well as our own experiments, are consistent with the idea that a greater initial brush thickness leads to a brush-solvent cushion that is more effective in separating the surfaces. It should also be borne in mind that under the same loading conditions, a smaller fraction of the film is compressed for films of higher initial thickness.

\subsection{Sliding Speed as a Parameter}

The 250-nm-thick P12MA in a brush-brush configuration $(0.1 \mathrm{~cm} / \mathrm{s}$ at $20 \mathrm{mN})$ was shown to be stable during tests lasting several days (sliding distance $>100 \mathrm{~m}$ both in hexadecane and EO500). Friction coefficients of around 0.01 were recorded during this period. Due to the apparently high stability of $250 \mathrm{~nm}$ coatings, studies with $140 \mathrm{~nm}$ coatings were performed, in order to facilitate the observation of sliding-speed effects. Figure 5 presents tribological durability measurements obtained for 140-nm-thick P12MA coatings studied under $20 \mathrm{mN}$ at various sliding speeds.

Sliding at the lowest of the four chosen speeds $(0.1 \mathrm{~cm} / \mathrm{s})$ in hexadecane led to rapid coating degradation (Fig. 5). Increasing the maximal sliding speed was found to increase the coating durability. Due to the stochastic nature of the wear processes occurring during acquisition of the sliding distance- $\mathrm{COF}$ curves, there is a degree of variability among samples; however, a consistent trend was observed (also with different tribometer configuration, see Supplementary Material), in which sliding at lower speeds led to more rapid failure- the lower the Sommerfeld number, the faster the coating degradation. Similarly, low viscosities (Fig. 4) also led to faster coating degradation. Even in the absence of significant hydrodynamic lift, 


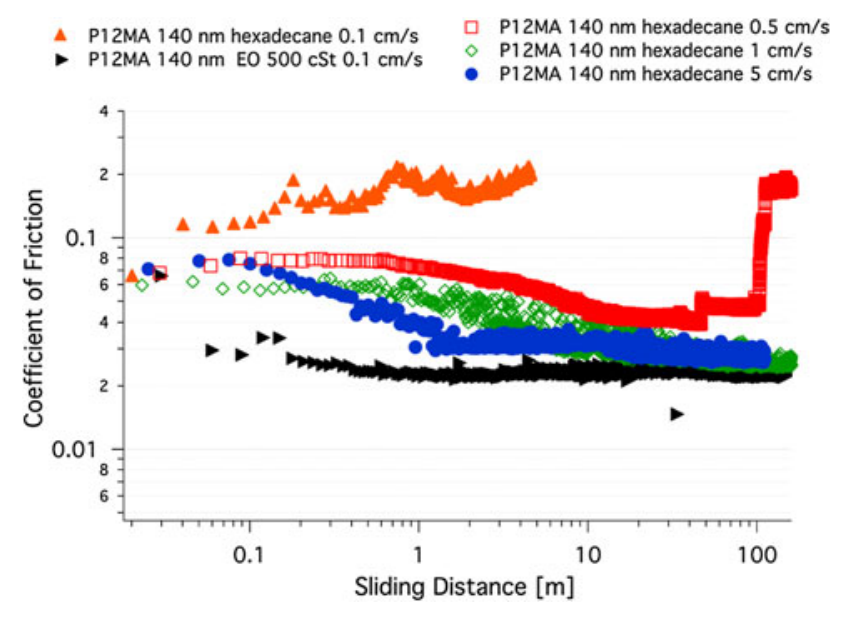

Fig. 5 Effect of sliding speed and base fluid on brush stability: Coefficient-of-friction evolution as a function of sliding distance in reciprocating motion of a silicon wafer and borosilicate glass balls, both sides modified with P12MA brushes (140 nm dry thickness)

velocity-dependent (i.e. time-dependent) effects come into play. Such effects can be explained by squeeze-film behaviour, analogous to the mechanism suggested by Hou et al. [29] for cartilage lubrication, and resistance to lubricant flow within the polymer, as postulated by Feiler et al. [30] for solvent within a polymer network, who reported up to a 1000 -fold increase in effective viscosity. At higher speed $\times$ viscosity values, the period of running-in prior to reaching constant sliding friction values appeared to be shorter. This may be a consequence of an initial 'brush-trimming' [26] or 'combing' [30] effect terminating more rapidly in the presence of greater hydrodynamic or viscous support.

\subsection{Load as a Parameter}

Stability tests at higher load $(80 \mathrm{mN}$, maximum Hertzian contact pressure ca $285 \mathrm{MPa}$ ) are presented only for $250 \mathrm{~nm}$ coatings, due to their superior stability under $20 \mathrm{mN}$ load (maximum Hertzian contact pressure ca $180 \mathrm{MPa}$ ) (Fig. 6). A symmetrically coated polymer-brushcoated tribological contact, when slid in hexadecane at $80 \mathrm{mN}$, revealed an initial coefficient of friction of 0.08 , which gradually decreased to 0.03 and then remained stable up to $>100 \mathrm{~m}$ sliding distance (see Supplementary Material for triboscopy).

An $80 \mathrm{mN}$ load contact in an ester oil of higher viscosity $\left(500 \mathrm{cSt}\right.$ at $20^{\circ} \mathrm{C}$ ) provided very low coefficient-of-friction values from the very first cycle (COF ca 0.006), similar to those obtained at $20 \mathrm{mN}$ load (Fig. 3). The lower coefficient-of-friction values recorded while sliding in EO50O compared to sliding in hexadecane may be the result of a number of factors, including: (a) the smaller degree of interpenetration of the opposing brush structures due to a greater hydrodynamic lift and possible squeeze-film effect

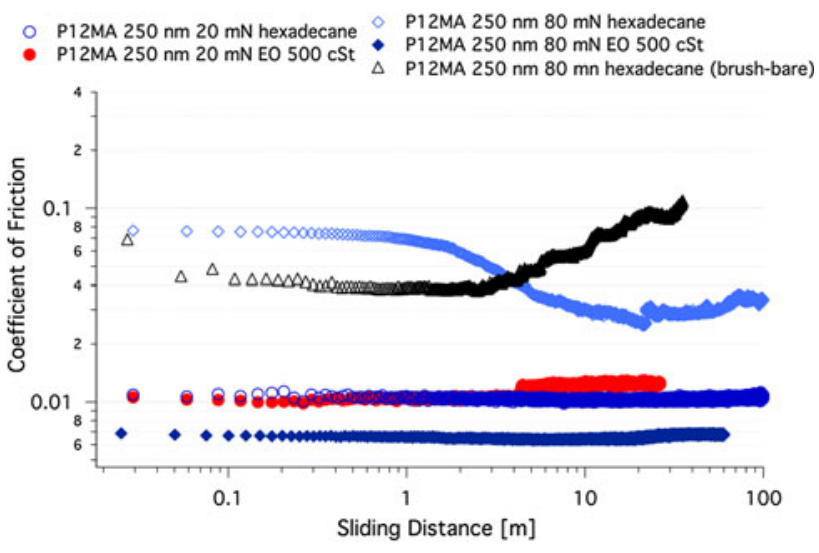

Fig. 6 Coefficient-of-friction evolution as a function of sliding distance in reciprocating motion of silicon wafer and borosilicate glass balls, both sides being modified with P12MA brushes $(250 \mathrm{~nm}$ dry thickness). The effects of applied load $(20$ and $80 \mathrm{mN})$ and lubricant on stability have been investigated. A control case of a $250 \mathrm{~nm}$-thick brush-coated silicon wafer slid against a bare ball under $80 \mathrm{mN}$ in hexadecane is also shown

and (b) smaller brush compressions occurring during sliding, due to the lower rate of 'squeeze-out' of the more viscous medium ( $500 \mathrm{cSt}$ vs $4 \mathrm{cSt}$ ) through the brush. The brush-brush configuration seems to be crucial for providing low-COF sliding and sustaining high loads-a singlesided, brush-bare configuration fails within the few first metres of sliding. However, it is interesting to observe that in the case of the $80 \mathrm{mN}$ experiments in hexadecane, the brush-bare friction was actually initially lower than the brush-brush friction, suggesting that in the latter case interpenetration was occurring, and leading to friction enhancement, as has been previously reported for dextran brushes [31]. The subsequent decrease of friction in the brush-brush case was, again, presumably due to brush trimming or combing.

\subsection{Extension to Substrates Other than Silicon}

UV/ozone-cleaned iron oxide samples were characterised with X-ray photoelectron spectroscopy (XPS) after a twostep initiator functionalisation protocol, in order to extract information on the approximate packing density of the initiating groups. Based on angle-resolved XPS data and organic overlayer thickness calculations, a coverage could be estimated as, on average, 2.7 nitrodopamine-based ATRP initiator molecules per $\mathrm{nm}^{2}$ of the substrate. This corresponds to a massive excess, when it is considered that dense PMMA brushes have maximal grafting densities of 0.7 chains $\mathrm{nm}^{-2}$ [23] and the current experiments involve a far bulkier monomer (more information on the XPS measurements is included in the Supplementary Material).

Iron oxide-coated silicon wafers were further functionalised with ca $250 \mathrm{~nm}$-thick P12MA polymer brushes, 


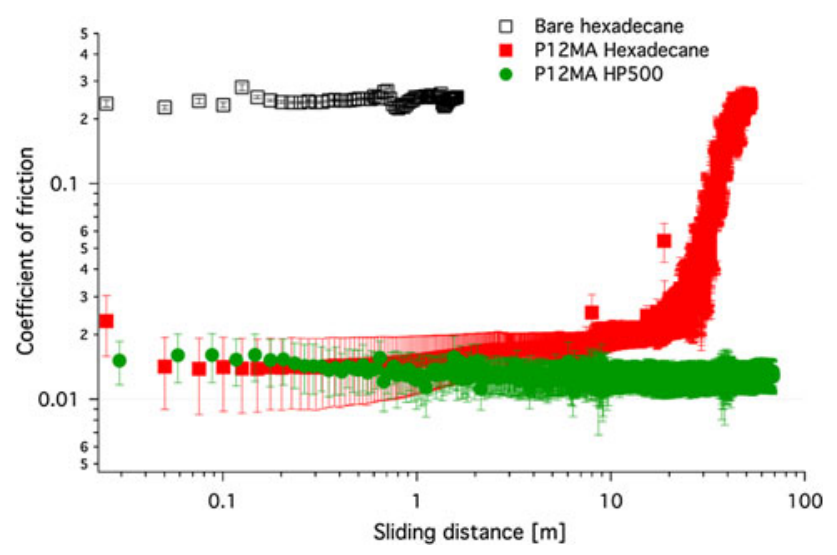

Fig. 7 Coefficient-of-friction evolution as a function of sliding distance in reciprocating motion of P12MA-brush-modified, iron oxide-coated wafers against P12MA-brush-coated 100Cr6 steel balls. Data obtained at $20 \mathrm{mN}$ load and $0.1 \mathrm{~cm} / \mathrm{s}$ sliding speed. Bare-bare system shown as a reference

synthesized with a $\mathrm{FeBr}_{2} / \mathrm{FeBr}_{3}$-based activator/deactivator system. These were studied in tribological contact against similarly functionalised $100 \mathrm{Cr} 6$ steel balls.

Applying the polymer coatings in this ball-on-disc configuration, a friction reduction by a factor of 10 was achieved, compared to the uncoated surfaces.

At $20 \mathrm{mN}$ load under hexadecane, a coefficient of friction below 0.02 was measured and maintained over a long sliding distance $(\approx 20 \mathrm{~m})$. When sliding took place in the more viscous EO500 oil, even longer sliding distances (>80 m) could be attained (Fig. 7).

As seen in Fig. 8, in the case of hexadecane-based sliding, the wear of the polymer coating progressed gradually, originating from the ends of the wear arc, where the speed values diminished to 0 prior to reversal of the direction of motion, and where the hydrodynamic forces are the lowest (see Supplementary Material). In contrast to the high-speed areas-in which the low $\mathrm{COF}$ was maintained over the longest distance- the low-speed regions, in the vicinity of turning points, showed faster brush degradation, presumably due to greater brush compression and interpenetration.

At asperity-asperity contacts, the high local pressure will enhance the probability of wear in thin polymeric coatings. By using more viscous liquids, or fabricating thicker polymeric films, the substrate asperities are more protected, and thus this effect can be reduced. Indeed, in the studied ironbased contact pair, enhanced durability was observed when using EO500 as a lubricant (Fig. 8) and no signs of degradation could be observed by optical microscopy.

\section{Conclusions}

A series of coatings based on poly(alkyl methacrylate) polymer brushes was synthesized and tribologically evaluated in various base fluids. Based on the frictional data, good and bad solvents were identified for each polymer and their further applicability for oil-based lubrication was assessed. It was shown that when tribologically stressed in a dry state or in bad solvents, the polymer coatings underwent rapid degradation-even in the initial stages of sliding. Polymer brushes sliding against each other in appropriate base fluids were shown to be capable of maintaining low coefficientof-friction values (on the order of 0.01) over thousands of reciprocating cycles ( $>100 \mathrm{~m}$ total distance).

Symmetrically P12MA-brush-functionalised surfaces sliding at low speeds in several different base fluids displayed low coefficients of friction that were almost independent of the speed $\times$ viscosity value. This behaviour
Fig. 8 Coefficient-of-friction evolution during sliding as a function of position along the sliding arc (expressed as sliding speed). Upper image displays triboscopy [32] of sliding in hexadecane; the onset of the coefficient-of-friction increase (correlated with coating removal) begins in the lowspeed regions. Lower image displays sliding in EO500 oil; measured coefficient-of-friction remains stable and low. The black arrows indicate time evolution

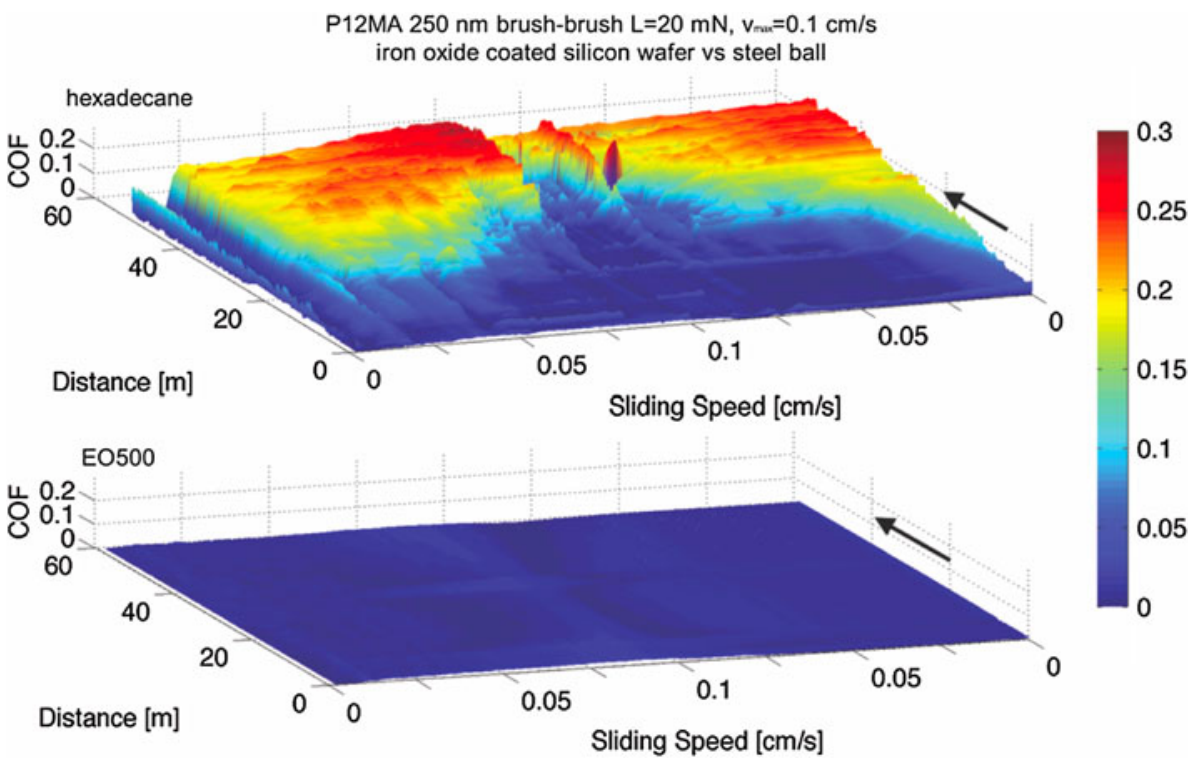


replaced the characteristic boundary and mixed regimes observed in the bare-bare configuration, and showed a transition directly into hydrodynamic lubrication at higher speeds.

The stability of the P12MA coating was evaluated at various coating thickness (chain length) values, lubricants, sliding speeds and applied loads. In general, P12MA coatings studied in a brush-brush configuration (on silicon wafers and borosilicate balls) displayed better wear resistance when the coating thickness was higher and/or when studied under conditions corresponding to higher Sommerfeld number-even when below the onset of the purely hydrodynamic regime, where the opposing brushes cease to interact. This can be attributed to a greater cushioning of the asperities by the brush, which is influenced by the polymer chain length, the amount of hydrodynamic lift itself (reducing the compression of the brush, and being promoted by lower load, a higher speed or higher viscosity), possible squeeze-film effects or the rate at which lubricant is pressed out from the brush-coated contact, which, in turn, is lower for more viscous lubricants.

The modification of iron oxide-coated silicon wafers and AISI52100 steel balls with catechol-based initiators and P12MA polymer, and subsequent achievement of low coefficient-of-friction values $(0.01-0.02)$ on such surfaces was successfully demonstrated, confirming the real engineering potential of lubrication by oil-compatible polymer brushes.

\section{References}

1. Klein, J., Kumacheva, E., Mahalu, D., Perahia, D., Fetters, L.J.: Reduction of frictional forces between solid-surfaces bearin polymer brushes. Nature 370, 634-636 (1994)

2. Klein, J., Kumacheva, E., Perahia, D., Mahalu, D., Warburg, S.: Interfacial sliding of polymer-bearing surfaces. Faraday Discuss. 98, 173 (1994)

3. Klein, J.: Shear of polymer brushes. Colloids Surf. A Physicochem. Eng. Aspects 86, 63-76 (1994)

4. Raviv, U., Giasson, S., Kampf, N., Gohy, J.-F., Jérôme, R., Klein, J.: Normal and frictional forces between surfaces bearing polyelectrolyte brushes. Langmuir 24(16), 8678-8687 (2008)

5. Schorr, P.A., Kwan, T.C.B., Kilbey, S.M., Shaqfeh, E.S.G., Tirrell, M.: Shear forces between tethered polymer chains as a function of compression, sliding velocity, and solvent quality. Macromolecules 36(2), 389-398 (2002)

6. Li, A., Benetti, E.M., Tranchida, D., Clasohm, J.N., Schönherr, H., Spencer, N.D.: Surface-grafted, covalently cross-linked hydrogel brushes with tunable interfacial and bulk properties. Macromolecules 44(13), 5344-5351 (2011)

7. Tsujii, Y., Nomura, A., Okayasu, K., Gao, W., Ohno, K., Fukuda, T.: AFM studies on microtribology of concentrated polymer brushes in solvents. J. Phys. Conf. Ser. 184(1), 1-7 (2009)

8. Limpoco, F.T., Advincula, R.C., Perry, S.S.: Solvent dependent friction force response of polystyrene brushes prepared by surface initiated polymerization. Langmuir 23(24), 12196-12201 (2007)
9. Landherr, L.J.T., Cohen, C., Agarwal, P., Archer, L.A.: Interfacial friction and adhesion of polymer brushes. Langmuir 27(15), 9387-9395 (2011)

10. Nomura, A., Okayasu, K., Ohno, K., Fukuda, T., Tsujii, Y.: Lubrication mechanism of concentrated polymer brushes in solvents: effect of solvent quality and thereby swelling State. Macromolecules 44(12), 5013-5019 (2011)

11. Sui, X., Zapotoczny, S., Benetti, E.M., Memesa, M., Hempenius, M.A., Vancso, G.J.: Grafting mixed responsive brushes of poly( $N$-isopropylacrylamide) and poly(methacrylic acid) from gold by selective initiation. Polym. Chem. 2, 879-884 (2011)

12. Patten, T.E., Matyjaszewski, K.: Atom transfer radical polymerization and the synthesis of polymeric materials. Adv. Mater. 10(12), 901-915 (1998)

13. Braunecker, W.A., Matyjaszewski, K.: Controlled/living radical polymerization: Features, developments, and perspectives. Prog. Polym. Sci. 32(1), 93-146 (2007)

14. Nomura, A., Ohno, K., Fukuda, T., Sato, T., Tsujii, Y.: Lubrication mechanism of concentrated polymer brushes in solvents: effect of solvent viscosity. Polym. Chem. 3(1), 148-153 (2012)

15. Bielecki, R.M., Benetti, E.M., Kumar, D., Spencer, N.D.: Lubrication with oil-compatible polymer brushes. Tribol. Lett. 45(3), 477-487 (2012)

16. Babu, K., Dhamodharan, R.: Grafting of poly(methyl methacrylate) brushes from magnetite nanoparticles using a phosphonic acid based initiator by ambient temperature atom transfer radical polymerization (ATATRP). Nanoscale Res. Lett. 3, 109-117 (2008)

17. Lu, G., Li, Y.-M., Lu, C.-H., Xu, Z.-Z.: Corrosion protection of iron surface modified by poly(methyl methacrylate) using surface-initiated atom transfer radical polymerization (SI-ATRP). Colloid Polym. Sci. 288(14-15), 1445-1455 (2010)

18. Fan, X.W., Lin, L.J., Dalsin, J.L., Messersmith, P.B.: Biomimetic anchor for surface-initiated polymerization from metal substrates. J. Am. Chem. Soc. 127, 15843-15847 (2005)

19. Gong, R., Maclaughlin, S., Zhu, S.: Surface modification of active metals through atom transfer radical polymerization grafting of acrylics. Appl. Surf. Sci. 254(21), 6802-6809 (2008)

20. Amstad, E., Gillich, T., Bilecka, I., Textor, M., Reimhult, E.: Ultrastable iron oxide nanoparticle colloidal suspensions using dispersants with catechol-derived anchor groups. Nano Lett. 9(12), 4042-4048 (2009)

21. Sanjuan, S., Perrin, P., Pantoustier, N., Tran, Y.: Synthesis and swelling behavior of $\mathrm{pH}$-responsive polybase brushes. Langmuir 23, 5769-5778 (2007)

22. Schmitz, T.L., Action, J.E., Ziegert, J.C., Sawyer, W.G.: The difficulty of measuring low friction: Uncertainty analysis for friction coefficient measurements. J. Tribol. Trans. Asme 127, 673-678 (2005)

23. Ishikawa, T., Kobayashi, M., Takahara, A.: Macroscopic frictional properties of poly(1-(2-methacryloyloxy)ethyl-3-butyl imidazolium bis (trifluoromethanesulfonyl)-imide) brush surfaces in an ionic liquid. ACS Appl. Mater. Interfaces 2(4), 1120-1128 (2010)

24. Öztürk, E., Turan, E., Caykara, T.: Fabrication of ultrahydrophobic poly(lauryl acrylate) brushes on silicon wafer via surfaceinitiated atom transfer radical polymerization. Appl. Surf. Sci. 257(3), 1015-1020 (2010)

25. Perry, S.S., Yan, X., Limpoco, F.T., Lee, S., Müller, M., Spencer, N.D.: Tribological properties of poly(L-lysine)-graft-poly(ethylene glycol) films: influence of polymer architecture and adsorbed conformation. ACS Appl. Mater. Interfaces 1(6), 1224-1230 (2009)

26. Dai, L., Minn, M., Satyanarayana, N., Sinha, S.K., Tan, V.B.: Identifying the mechanisms of polymer friction through molecular dynamics simulation. Langmuir 27, 14861-14867 (2011)

27. Zhang, Z., Morse, A.J., Armes, S.P., Lewis, A.L., Geoghegan, M., Leggett, G.J.: Effect of brush thickness and solvent composition on the friction force response of poly(2-(methacryloyloxy) ethylphosphorylcholine) brushes. Langmuir 27, 2514-2521 (2011) 
28. Sakata, H., Kobayashi, M., Otsuka, H., Takahara, A.: Tribological properties of poly(methyl methacrylate) brushes prepared by surface-initiated atom transfer radical polymerization. Polym. J. 37(10), 767-775 (2005)

29. Hou, J.S., Mow, V.C., Lai, W.M., Holmes, M.H.: An analysis of the squeeze-film lubrication mechanism for articular-cartilage. J. Biomech. 25(3), 247-259 (1992)

30. Feiler, A., Plunkett, M.A., Rutland, M.W.: Atomic force microscopy measurements of adsorbed polyelectrolyte layers. 1 .
Dynamics of forces and friction. Langmuir 19(10), 4173-4179 (2003)

31. Rosenberg, K.J., Goren, T., Crockett, R., Spencer, N.D.: Loadinduced transitions in the lubricity of adsorbed poly(L-lysine)-gdextran as a function of polysaccharide chain density. ACS Appl. Mater. Interfaces 3(8), 3020-3025 (2011)

32. Belin, M., Lopez, J., Martin, J.M.: Triboscopy, a quantitative tool for the study of the wear of a coated material. Surf. Coat. Technol. 70(1), 27-31 (1994) 